\title{
Clinico-pathological Profile and Immediate Hospital Outcome of Acute Pancreatitis
}

\author{
Md. Ishtiaq Alam ${ }^{*}$, Md. Mujibur Rahman², Ahmedul Kabir ${ }^{3}$, Md. Uzzwal Mallik ${ }^{4}$, Saima Azad ${ }^{5}$
}

\begin{abstract}
Abstruct:
Background: Acute pancreatitis (AP) is a significant cause of acute abdominal pain, morbidity and hospitalization. Knowing the details of the clinico-pathological presentation and outcome of the disease may aid the better understanding of the disease.

Objective: The study was designed to assess the clinico-pathological profile and to estimate immediate hospital outcome of acute pancreatitis patients admitted into a tertiary care hospital.

Materials and methods: This hospital based cross-sectional type of observational study was conducted at Dhaka Medical College Hospital (DMCH), for one-year period (June 2018-May 2019). Total 106 established cases of acute pancreatitis were included in this study. Data was analyzed by the SPSS 22 Windows version and graph \& chart were expressed by using SPSS 22 and MS Excel. The result was presented with tables and charts.

Results: Among 106 patients, mean age of the patients was $42.5 \pm$ I I.3 SD (years), with male predominance (male: female-1.2:I).All of the patients complained abdominal pain (100\%) while anorexia (48.1\%), 43.4\% had vomiting and $31.1 \%$ had fever. Regarding clinical signs, abdominal distension (45.3\%), paralytic ileus (34.9\%) and dehydration (43.4\%) were found to be the major clinical signs. The common location of pain was in epigastric region (73.6\%) with radiation to back in $20.8 \%$ patients. Pain was severe agonizing in nature in $76.4 \%$ cases. Of all, gall stone disease (17.0\%) was the most prevalent cause. According to APACHE-II score, $74.5 \%$ patients had mild pancreatitis (APACHE-II <8) while according to Glasgow criteria, $66.0 \%$ had mild disease (Glasgow score <3). Mean APACHE-Il score was $7.07 \pm 2.50$ and Glasgow score was 2.90 2 2.33. Mean hospital stays were $8.9 \pm 3.6$ days and NPO (nothing per oral) $4.9 \pm 1.9$ days. Among the patients, $77.4 \%$ patients were alive and mortality rate was $22.6 \%$.

Conclusion: Although, a larger portion of the patients had no identifiable cause but gall stone was the most common etiology. Pain commonly located in epigastric region with radiation to back. Of all the cases, more than sixty percent of the patients had mild pancreatitis estimated by APACHE II score and Glasgow score. Mean hospital stays were about 9 days and nothing per oral was about 5 days. Overall mortality rate was $22.6 \%$.
\end{abstract}

Key words: Acute pancreatitis, Clinico-pathological profile, Gallstone pancreatitis, Hospital outcome.

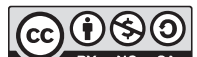
Copyright: (C) 2022Alam MI. This is an open access article published under the Creative Commons Attribution-NonCommercial-NoDerivatives 4.0 International License, which permits use, distribution and reproduction in any medium, provided the original work is properly cited, is not changed in any way and it is not used for commercial purposes.

Received: 04 March, 202I;

Accepted: 30 November, 2021

1. Medical Officer, National Institute of Cardiovascular Diseases and Hospital, Dhaka

2. Professor, Department of Medicine, Bangabandhu Sheikh Mujib Medical University

3. Professor of Medicine and ADG (Admin), Directorate General of Health Services, Mohakhali, Dhaka

4. Assistant professor, Department of Medicine, Dhaka Medical College Hospital, Dhaka

5. Indoor Medical Officer, Department of Medicine, Dhaka Medical College Hospital, Dhaka

*Corresponding author: Dr. Md. Ishtiaq Alam, Medical Officer, National Institute of Cardiovascular Diseases and Hospital, Dhaka, email-ishtiaqalamelite@gmail.com

\section{Introduction:}

Acute pancreatitis describes an acute inflammatory process of the pancreas that can range from mild interstitial pancreatitis to severe pancreatitis with pancreatic necrosis and concomitant multi-organ failure. Acute pancreatitis is typically rapid in onset and most commonly encountered in its mild to moderate form ${ }^{1}$. The diagnosis of acute pancreatitis usually requires a combination of clinical, laboratory and radiological findings. A number of international guidelines have suggested two of the following three features are required for the diagnosis: (i) abdominal pain consistent with acute pancreatitis (acute onset of persistent severe epigastric 
pain often radiating to the back), (ii) serum lipase activity (or amylase activity) at least three times greater than the upper limit of normal ${ }^{2}$ and iii) characteristic findings of acute pancreatitis on abdominal ultrasound (a CT scan or MRI is considered if the diagnosis is uncertain $)^{3}$. Amylase and lipase are released from acinar cells during acute pancreatitis and their concentration in the serum is used to confirm diagnosis. Serum amylase concentrations exceeding three times the normal upper limit support the diagnosis of acute pancreatitis. Amylase concentrations generally rise in the serum within a few hours after the onset of symptoms and return to normal values within 3-5 days ${ }^{4}$. However, amylase activity might remain within normal range on admission in $19 \%$ of the patients ${ }^{5}$. Conventional abdominal ultrasound scan should be performed in every patient. Plain radiograph finding may range from no abnormalities in mild disease to localized ileus of a segment of small intestine ("sentinel loop") or the colon cut-off sign in more severe disease. Imaging is used to assess severity of acute pancreatitis by using Computed Tomography (CT) grading system of Balthazar and the CT Severity Index (CTSI). Severity of acute pancreatitis is linked to the presence of systemic organ dysfunctions and/or necrotizing pancreatitis ${ }^{6}$. Though the exact pathogenesis of acute pancreatitis remains elusive, many theories have been proposed like abnormal biliopancreatic duct common pathway theory, pancreatic auto digestion theory, gallstone migration theory, enzyme activation theory, kinin and complement activation theory, microcirculation disturbance theory, and pancreatic acinar cell apoptosis and necrosis theory. In a study conducted by VineetChoudhary et al. ${ }^{7}$ found that increase of serum lipase was better diagnostic tool. Blood sugar $>200 \mathrm{mg} \%$, serum calcium $<8 \mathrm{mg} \%$ were important poor prognostic indicators ${ }^{7}$.By raising the cut off level to $1000 \mathrm{IU} / 1$ (more than three times then the normal limit) serum amylase has a specificity approaching $95 \%$, but a sensitivity as low as $61 \%$ in some studies. In case of lipase, at a cut off activity of 600 IU/1, most studies have reported specificities above 95\%, with sensitivities ranging between $55 \%$ and $100 \%{ }^{8}$. Local complications of acute pancreatitis are ${ }^{9}$-acute peri-pancreatic fluid collections, pancreatic pseudocysts, acute necrotic collections, walled-off pancreatic necrosis (WON). Systemic consequences are ${ }^{10}-1$. Priming of neutrophils, 2. Endotoxemia, 3. Bacterial translocation, 4. Impaired systemic immunity, 5. Multiorgan failure. Most episodes of acute pancreatitis are mild and self-limiting, needing only brief hospitalization. However, $20 \%$ of patients develop a severe disease with local and extra-pancreatic complications characterized by early development and persistence of hypovolemia and multiple organ dysfunctions.

\section{Materials and Methods:}

This study was done in Medicine Department of Dhaka Medical College Hospital for one year (Jun 2018-May 2019). Informed written consent from the patient or relatives were taken. It was a Cross-sectional type of observational study. Consecutive sampling was used. Total 106 patients were included in the study. Severity classification of acute pancreatitis was defined as follows- Mild: AP without organ failure (OF), Moderate: AP without OF, but with local complications, Severe: Early severe acute pancreatitis (SAP)A. Fulminant: OF within 72 hours, B. Sub fulminant: OF within 4-7 days. Late SAP - OF or infected pancreatic necrosis (IPN) after 7 days. Critical AP: Persistent OF + IPN. Following admission, patient diagnosed as acute pancreatitis according to Atlanta classification and patients were classified into mild, moderate and severe acute pancreatitis and Glasgow scoring, CT severity index (CTSI), complete hemogram were done for all the patients and Contrast-enhanced computed tomography (CECT) abdomen was done when indicated. After confirmation all the AP cases were selected in according to the inclusion and exclusion criteria. Statistical significance was set as $95 \%$ confidence level at 5\% acceptable error level. Patients' characteristics were reported as percentages or mean \pm standard deviation when variables were continuous while categorical variables were expressed with count (percentage). Statistical analysis was done by Chi Squared test and t-test. Data was analyzed by the SPSS 22 Windows version and graph \& chart were expressed by using SPSS 22 and MS Excel. The result was presented with tables and charts.

\section{Result:}

Among 106 patients, mean age was $42.5 \pm 11.3$ years. Highest age was 68 years and minimum age was 22 years. Majority of the patients $(40.6 \%)$ were from age group 31-40 years (Table 1).

Table 1 : Age distribution of study population $(N=106)$

\begin{tabular}{lc}
\hline Age $($ Years $)$ & Percentage \\
\hline $18-30$ years & 8.5 \\
$31-40$ years & 40.6 \\
$41-50$ years & 28.3 \\
$51-60$ years & 14.2 \\
$>60$ years & 8.5 \\
\hline
\end{tabular}


Among the study participants, $54.7 \%$ patients were male and $45.3 \%$ were female with male to female ratio was $1.2: 1$. All of the patients complained abdominal pain. $43.4 \%$ patients had vomiting and $31.1 \%$ had fever. (Table 2 ) The common location of pain was in epigastric region $(73.6 \%)$ with radiation to back in $20.8 \%$ patients. Pain was severe agonizing in nature in $76.4 \%$ cases. (Table 3 )

Table 2: Clinical profile of patients with acute pancreatitis of study population $(N=106)$.

\begin{tabular}{|c|c|c|}
\hline Clinical features & Erequency (n) & Percentage $(\%)$ \\
\hline \multicolumn{3}{|l|}{ Symptoms } \\
\hline Abdominal pain & 106 & 100 \\
\hline Vomiting & 46 & 43.4 \\
\hline Fever & 33 & 31.1 \\
\hline Nausea & 18 & 17.0 \\
\hline Anorexia & 51 & 48.1 \\
\hline \multicolumn{3}{|l|}{ Signs } \\
\hline Abdominal tenderness & 106 & 100 \\
\hline Anaemia & 61 & 57.5 \\
\hline Abdomnal distension & 48 & 45.3 \\
\hline Dehydration & 46 & 43.4 \\
\hline Paralytic ileus & 37 & 34.9 \\
\hline Pleural effusion & 31 & 29.2 \\
\hline Jaundice & 19 & 17.9 \\
\hline Respiratory distress & 18 & 17.0 \\
\hline
\end{tabular}

Table 3: Character of abdominal pain in the studied patients $N=106)$.

\begin{tabular}{lcc}
\hline Abdominal pain & $\begin{array}{c}\text { Frequency } \\
(\mathrm{n})\end{array}$ & $\begin{array}{c}\text { Percentage } \\
(\%)\end{array}$ \\
\hline Location & 78 & 73.6 \\
$\quad$ Epigastric & 28 & 26.4 \\
$\quad$ Diffuse & & \\
Character & 81 & 76.4 \\
$\quad$ Severe agonizing & 25 & 23.6 \\
Dull aching & 22 & 20.8 \\
$\quad$ Radiation to back & 57 & 53.8 \\
Relieved by forward bending & 52 & 49.1 \\
$\quad$ Exacerbated by taking heavy meal & 52 \\
Total duration of pain: & $3.88 \pm 1.55$ \\
$\quad$ Mean \pm SD (days) & \multicolumn{2}{c}{} \\
\hline
\end{tabular}

Most common cause was gall stone disease (17.0\%). This was followed by alcohol (12.3\%) and Post-Endoscopic retrograde cholangiopancreatography (ERCP) status (8.5\%). (Figure 1) In 53.8\% cases, no cause was found (idiopathic).

\section{Causes of acute pancreatitis}

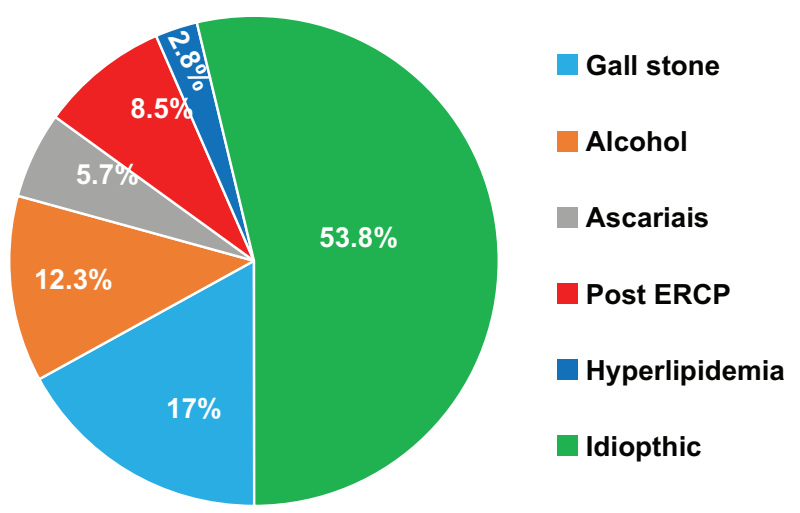

Figure 1: Causes of acute pancreatitis in study population $(n=106)$.

Laboratory findings of study population showed that, all the patients had leukocytosis and raised C-reactive protein. Hypocalcemia was prevalent in $34 \%$ patients (Table 4).

Table 4: Laboratory findings in patients with acute pancreatitis $(N=106)$

\begin{tabular}{lc}
\hline Lab parameters & Value \\
\hline Mean amylase (IU/L) & $577.12 \pm 292.92$ \\
Mean lipase (IU/L) & $464.04 \pm 303.26$ \\
CRP (mg/L) & $113.84 \pm 44.91$ \\
Raised & $106(100 \%)$ \\
Mean calcium (mg/dL) & $8.66 \pm 1.28$ \\
Hypocalcemia & $36(34.0 \%)$ \\
Normal Calcium & $70(66.0 \%)$ \\
Leukocytosis & $106(100 \%)$ \\
\hline
\end{tabular}

According to APACHE-II score, $74.5 \%$ patients had mild pancreatitis (APACHE-II $<8$ ) while according to Glasgow criteria, $66.0 \%$ had mild disease (Glasgow score $<3$ ). Mean APACHE-II score was $7.07 \pm 2.50$ and Glasgow score was $2.90 \pm 2.33$. (Figure 2) Total duration of hospital stays were found 3 to 16 day, and nothing per oral was found 2 to 10 days. (Table 5) Complications of acute pancreatitis such as liver dysfunction, renal dysfunction $17.9 \%, 18.8 \%$ respectively and pneumonia $14.2 \%$, pseudocyst $8.4 \%$. (Table 6) 


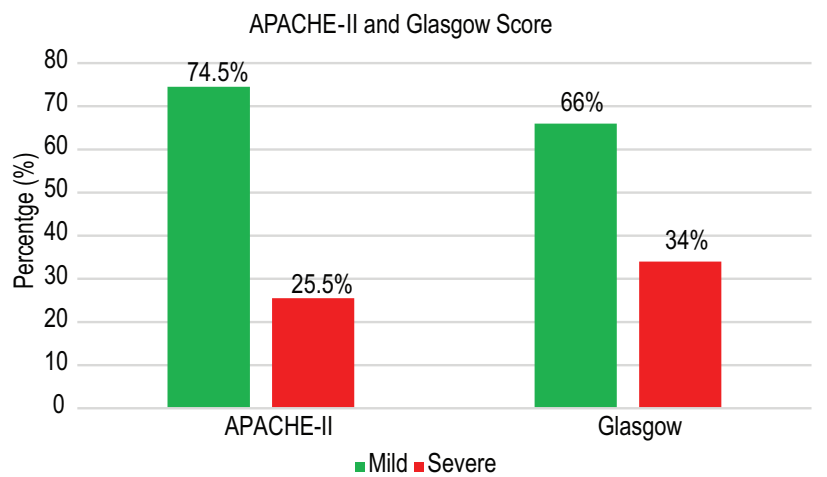

Figure 2: Severity of acute pancreatitis according to APACHE-II and Glasgow scoring criteria within 24 hours of admission $(n=106)$.

Among 106 patients $77.4 \%$ patients were alive and mortality rate was $22.6 \%$ and $38.9 \%$ patients died due to multi-organ system failure and $27.8 \%$ due to sepsis, $16.7 \%$ due to ARDS. (Figure 3)

Table 5 : Clinical course in acute pancreatitis $(N=106)$.

\begin{tabular}{lccc}
\hline Clinical course and outcome & Mean & $\begin{array}{c}\text { Std. } \\
\text { Deviation }\end{array}$ & $\begin{array}{c}\text { Min- } \\
\text { Max }\end{array}$ \\
\hline Hospital stays (days) & 8.97 & 3.65 & $3-16$ \\
NPO (days) & 4.89 & 1.92 & $2-10$ \\
Time to alleviation ofsymptoms & 3.89 & 1.65 & $1-7$ \\
(days) & & & \\
\hline
\end{tabular}

*NPO-Nothing per oral

Association between APACHE-II and outcome of the patients with acute pancreatitis showed that death was significantly more $(\mathrm{p}<0.001)$ prevalent in patients with APACHE-II $\geq 8$. Association between Glasgow score and outcome of the patients with acute pancreatitis showed that death was significantly more $(p<0.001)$ prevalent in patients with Glasgow score $\geq 3$. (Table 7)

Table 6: Complications of study population $(N=106)$

\begin{tabular}{lcc}
\hline Characters & Number & Percentage \\
\hline Renal dysfunction & 20 & 18.8 \\
Liver dysfunction & 19 & 17.9 \\
ARDS & 18 & 17.9 \\
Sepsis & 18 & 17.0 \\
Pneumonia & 15 & 14.2 \\
Shock & 12 & 11.0 \\
Pseudocyst & 9 & 8.4 \\
\hline
\end{tabular}

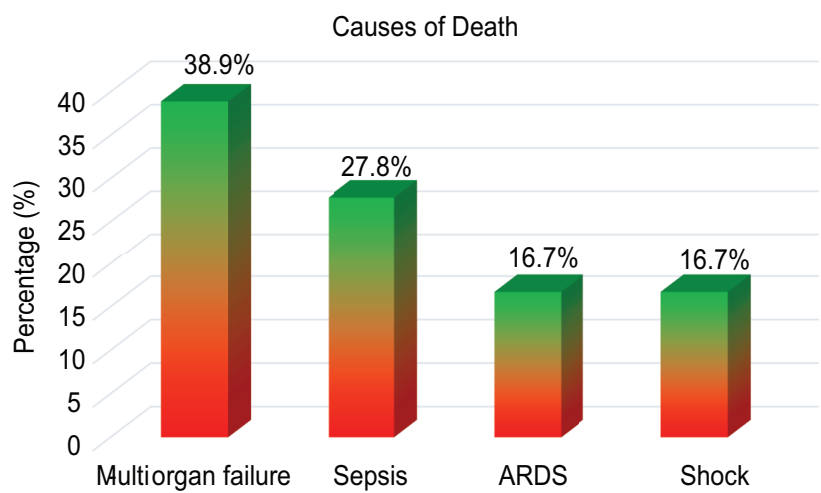

Figure 3: Causes of death due to acute pancreatitis of our study population $(N=24)$.

Table 7 : Association between APACHE-II and Glasgow score with outcome of patients with acute pancreatitis $(N=106)$.

\begin{tabular}{lccc}
\hline APACHE-II & \multicolumn{2}{c}{ Outcome } & $\begin{array}{c}\text { P- } \\
\text { value }\end{array}$ \\
\cline { 2 - 3 } & Alive & Death & $<0.001$ \\
\hline 8 (Mild) & N79 $(100 \%)$ & 0 & \\
ed 8 (Severe) & N9 $(33.3 \%)$ & N18 $(66.7 \%)$ & \\
Total & N88(100\%) & N18 $(100 \%)$ & \\
Glasgow score & N70(100\%) & 0 & 0.001 \\
$<3$ (Mild) & & & \\
$\geq 3$ (Severe) & N18(50\%) & N18(50\%) & \\
\hline Total & N88(83\%) & N18 $(17 \%)$ & \\
\hline
\end{tabular}

Mean serum amylase and CRP level was significantly higher in dead patients $(p=0.003$ and $<0.001)$ with acute pancreatitis. Serum lipase level is higher in dead patients but the study was nonsignificant $(\mathrm{p}=0.166)$. Mean serum calcium level was significantly higher in alive patients than dead $(\mathrm{p}<0.001)$. (Table 8$)$

Table 8: Association between mean serum biochemical parameters and outcome of patients with acute pancreatitis $(n=106)$.

\begin{tabular}{lllr}
\hline Biochemical parameters & \multicolumn{2}{c}{ Outcome } & P- \\
\cline { 2 - 2 }$($ Mean \pm SD) & Alive & Death & value \\
\hline
\end{tabular}

\begin{tabular}{llll}
\hline Serum amylase (IU/L) & $538.86 \pm 276.98$ & $764.14 \pm 304.53$ & 0.003
\end{tabular}

Serum lipase (IU/L) $\quad 445.55 \pm 306.89554 .40 \pm 275.05 \quad 0.166$ $\mathrm{CRP}(\mathrm{mg} / \mathrm{L}) \quad 102.12 \pm 38.58 \quad 171.11 \pm 25.62<0.001$

\begin{tabular}{llll} 
Serum calcium $(\mathrm{mg} / \mathrm{dL})$ & $9.03 \pm 1.01$ & $6.82 \pm 0.76$ & $<0.001$ \\
\hline
\end{tabular}

Multivariate regression analysis of different risk factors for developing acute pancreatitis was done. All the risk factors which had a significance level of $p<0.05$ in univariate analysis was included. Age was found to be independently associated with death in the study population ( $\mathrm{p}=0.013)$. Age had OR of $1.087(95 \%$ CI 1.018-1.160) . (Table 9) 
Table 9 : Multivariate analysis of risk factors for death $(N=106)$.

\begin{tabular}{lccc}
\hline Variables & Odds ratio & $95 \%$ CI & p-value \\
\hline Age (years) & 1.087 & $1.018-1.160$ & 0.013 \\
Hospital stays (days) & 1.087 & $0.515-2.294$ & 0.827 \\
Time to alleviation of & 1.457 & $0.320-6.640$ & 0.626 \\
symptoms (days) & & & \\
Serum amylase (IU/L) & 1.001 & $0.999-1.003$ & 0.426 \\
\hline
\end{tabular}

\section{Discussion:}

Acute pancreatitis is an inflammatory process of the pancreas with varying involvement of regional tissues or remote organ systems ${ }^{11}{ }^{12}$. Acute pancreatitis (AP) is a relatively common disease with incidence of 5-80 per 1,00,000 members of the population and the number of new cases has shown a steady increase in recent years ${ }^{13}$. Most patients with AP show spontaneous resolution without complications; however, 10 $\%-20 \%$ of patients experience a severe attack with increased risk of mortality up to $25 \%{ }^{14}$.

In this study, the age range of patient was 22 to 68 years with a peak incidence is in the fourth decade (31-40 years) followed by $41-50$ years $(28.3 \%)$. Mean age was $42.5 \pm 11.3$ years. This is comparable to the studies done by Raghu $\mathrm{M} \mathrm{G}$ et al. and Negi et al. where it was $42.9 \pm 15.9$ years and $42.89 \pm 12.53$ years respectively ${ }^{15}{ }^{16}$. In our country the lower age incidence of acute pancreatitis may be due to lower life expectancy of Bangladeshi people, occurrence of gallstone disease in the female in early age due to early marriage and multiple pregnancies. Study data showed that ascariasis, mumps and trauma are common aetiological factors that more commonly affect younger age group ${ }^{17}$. Males $(54.7 \%)$ were predominant than females (45.3\%) and the male to female ratio was 1.2:18 19.

Abdominal pain is the cardinal symptom of acute pancreatitis. It occurs in $95 \%$ of cases and usually it is generalized to upper abdomen ${ }^{20}$. All of the patients in this study complained abdominal pain, especially epigastric pain (73.6\%). Among them, $20.8 \%$ had radiation to back. Pain exacerbated by taking heavy meal in $49.1 \%$ cases and in $53.8 \%$ cases the pain was relieved by forward bending. Jeffrey $\mathrm{CF}$ also described similar characters of pain in acute pancreatitis.

Regarding clinical signs, abdominal distension was found in $45.3 \%$ cases, $34.9 \%$ had paralytic ileus, $43.4 \%$ had dehydration and $48.1 \%$ had anorexia. Peri-pancreatic spread of inflammatory process may cause generalized ileus leading to distended abdomen ${ }^{20}$. Most common cause of acute pancreatitis was gall stone disease $(17.0 \%)$ which was followed by alcohol (12.3\%), post-ERCP complication ( $8.5 \%$ ) and ascariasis (5.7\%) cases. Hyperlipidemia was found in $2.8 \%$ cases. In $53.8 \%$ cases, no cause could be found (idiopathic). This finding was nearly similar to the study conducted by Ahmed KU et al. In their study entitled "Clinical profile of acute pancreatitis in a teaching hospital". The high incidence of idiopathic acute pancreatitis in our country is due to higher prevalence of infections because of poor nutrition, poor sanitation, overcrowding and lack of knowledge.

Serum amylase measurement showed that the mean value was $577.12 \pm 292.92 \mathrm{IU} / \mathrm{L}$ which is many times higher than normal. Similarly, mean serum lipase level was $464.04 \pm 303.26$ $\mathrm{IU} / \mathrm{L}$ which is also higher than normal value. CRP was raised in $100 \%$ cases. It is an acute phase protein, raised in many condition eg. Major trauma, sepsis, acute pancreatitis. CRP is of no value in the diagnosis of acute pancreatitis but sharply rises in pancreatic necrosis and that is a useful early marker of severity of pancreatitis ${ }^{21}$. CRP levels $>150 \mathrm{mg} / \mathrm{L}$ at 48 hours predict disease severity. Serum calcium levels fall in about $34 \%$ of cases, and that signifies severe pancreatitis. Association between Glasgow score and APACHE-II score with outcome of the patients showed that death was significantly more prevalent in high APACHE-II and high Glasgow score. Mean hospital stay was $8.97 \pm 3.65$ days with mean NPO 4.89 \pm 1.92 days. The longer the hospital stay and NPO, the severe the disease. Clinical outcome showed that, $22.6 \%$ of our patients were died in spite of treatment among whom; the major cause of death was organ failure in $38.9 \%$ cases and sepsis in $27.8 \%$ patients. But acute pancreatitis has an overall low mortality, of approximately $1 \%{ }^{22}$.

The risk of death increases with age, co-morbidities, and severe disease; in a recent meta-analysis, the risk of death was the highest among patients with both organ failure and infected necrosis ${ }^{23}$. In this study $22.6 \%$ people died. This is approximately double the findings of Ahad A. and colleagues ${ }^{17}$.

\section{Limitation}

This was a single-center study seen; no follow-up and only in-hospital outcome was considered and less scope for details investigations.

\section{Conclusion:}

Acute pancreatitis patients most commonly present with abdominal pain, abdominal distension along with anorexia. Although a larger portion of the patients had no identifiable cause but gall stone was the most common etiology. Pain commonly located in epigastric region with radiation to back. Of all the cases, more than sixty percent of the patients had 
mild pancreatitis estimated by APACHE II score and Glasgow score. Mean hospital stays were about 9 days and nothing per oral was about 5 days. Overall mortality rate was $22.6 \%$.

\section{References}

1. VanWoerkom R and Adler DG. Acute Pancreatitis: Review and Clinical Update, Hospital physician 2009; 45( 1): 9-19.

2. Basnayake $\mathrm{C}$ and Ratnam D. Blood tests for acute pancreatitis, Australian Prescriber 2015;38(4):128-130.

3. Banks PA, Bollen TL, Dervenis C, Gooszen HG, Johnson CD, Sarr MG. Classification of acute pancreatitis-2012: Revision of the Atlanta classification and definitions by international consensus, Gut2013;62(1):102-111.

4. Frossard JL, Steer ML and Pastor CM. Acute pancreatitis, Lancet 2008; 371:143-52.

5. Clavien PA, Robert J, Meyer P, Borst F, Hauser H, Herrmann F, Dunand V and Rohner A. Acute pancreatitis and normoamylasemia. Not an uncommon combination, Annals of Surgery $1989 ; 210(5): 614-20$.

6. Vengadakrishnan K and Koushik AK. A study of the clinical profile of acute pancreatitis and its correlation with severity indices, International Journal of Health Science 2015;9(4):410-17.

7. Choudhary V, Shekhawat N and Kumari N. Clinicopathological study of acute pancreatitis: a prospective study of 30 cases, International Surgery Journal 2015;2(2):19194.

8. Yadav D. A critical evaluation of laboratory tests in acute pancreatitis, The American Journal of Gastroenterology 2002;97(6):1309-31.

9. Greenberg JA, Hsu J, Bawazeer M, Marshall J, Friedrich JO, Nathens A, Coburn N, May GR, Pearsall E and McLeod RS. Clinical practice guideline: Management of acute pancreatitis, Canadian Journal of Surgery,2016; 59(2):12840.

10. Beger HG, \& Rau BM. Severe acute pancreatitis: Clinical course and management, World Journal of Gastroenterology 2014; 13(38):5043-51.

11. Baig SJ, Rahed A, \& Sen S. 2008, A prospective study of the aetiology, severity and outcome of acute pancreatitis in Eastern India. Tropical gastroenterology/ : official journal of the Digestive Diseases Foundation 2008;29(1):20-2.

12. Simchuk EJ, Traverso LW, Nukui Y and Kozarek RA. 2000, Computed tomography severity index is a predictor of outcomes for severe pancreatitis, American Journal of Surgery 2000;179(5):352-55.

13. Corfield AP, Cooper MJ, \& Williamson RCN. Acute pancreatitis: A lethal disease of increasing incidence, Gut 1985;26(7):724-29.
14. Yeung YP, Lam BYK \& Yip AWC APACHE system is better than Ranson system in the prediction of severity of acute pancreatitis. Hepatobiliary \& pancreatic diseases international/ : HBPD INT 2006;5(2):294-99.

15. Negi N, Mokta J, Sharma B, Sharma R, Jhobta A, Bodh V \& Ranjan A. Clinical profile and outcome of acute pancreatitis: A hospital-based prospective observational study in subhimalayan state', Journal of Association of Physicians of India 2018b; 66: 22-24.

16. Raghu MG, Wig JD, Kochhar R, Gupta D, Gupta R, Yadav TD, Agarwal R, Kudari AK, Doley RP \& Javed A. Lung complications in acute pancreatitis, Journal of the Pancreas 2007;8(2)177-85.

17. Ahmed KU, Ahad MA, Alim MA, \& Ekram AS. Clinical profile of acute pancreatitis in a teaching hospital, Bangladesh Medical Journal Khulna 2017;49 (1-2):7-12.

18. Besselink MGH, Verwer TJ, Schoenmaeckers EJP, Buskens E, Ridwan BU, Visser MR, Nieuwenhuijs VB, \& Gooszen HG. Timing of surgical intervention in necrotizing pancreatitis, Archives of Surgery, 2007;142(12):1194-1201.

19. Khanna AK, Meher S, Prakash S, Tiwary SK, Singh U, Srivastava A, \& Dixit VK Comparison of Ranson, Glasgow, MOSS, SIRS, BISAP, APACHE-II, CTSI Scores, IL-6, CRP, and procalcitonin in predicting severity, organ failure, pancreatic necrosis, and mortality in acute pancreatitis, HPB Surgery 2013;2013:1-10.

20. Cappell MS. Acute Pancreatitis: Etiology, Clinical Presentation, Diagnosis, and Therapy, Medical Clinics of North America 2008;92(4): 889-923.

21. Hasan M, Laila S, \& Mamun M. Clinical Pattern and Management of Acute Pancreatitis-Our Experience, Journal of Bangladesh College of Physicians and Surgeons 2014;31(3):122-27.

22. Peery AF, Dellon ES, Lund J, Crockett SD, McGowan CE, Bulsiewicz, WJ. Burden of Gastrointestinal Disease in the United States: 2012 Update, Gastroenterology 2012;143(5):1179-87.

23. Petrov MS, Shanbhag S, Chakraborty M, Phillips ARJ \& Windsor JA. Organ failure and infection of pancreatic necrosis as determinants of mortality in patients with acute pancreatitis, Gastroenterology. Elsevier Inc. 2010;139(3):813-20.

24. Ahad MA. Acute pancreatitis- a clinical update, Bangladesh Medical Journal Khulna 2017;50:35-40.

25. Knaus WA, Draper EA, Wagner DP, Zimmerman JE. APACHE II: a severity of disease classification systemÕ, Crit Care Med 1985;13:818-29. 